\title{
Bernoulli-Deng Equation and Its General Integral
}

\author{
Xingju Dang ${ }^{1}$, Xiaopan $\mathrm{Li}^{2}$, and Wenliang $\mathrm{Wu}^{3, *}$ \\ ${ }^{1,2,3}$ College of Physics \& Electronic Information Engineering, Zhaotong University, Zhaotong Yunnan, 657000, China \\ ${ }^{*}$ Corresponding author
}

\begin{abstract}
If real constants $\alpha, \beta$ (at least one is not zero) let $g(y) \mathrm{d}(f(y) / g(y)) / \mathrm{d} y=\alpha+\beta f / g$, then the naming the equation $\mathrm{d} y / \mathrm{d} x=P(x) f(y)+Q(x) \mathrm{g}(y)$ for Bernoulli-Deng Equation is suggested. The equation can be integrated and its general integral is given.
\end{abstract}

Keywords-Bernoulli equation; general integral; generalized Bernoulli equation

\section{INTRODUCTION}

In $1695, \mathrm{~J}$. Bernoulli proposed the question of solution of equation

$$
\frac{\mathrm{d} y}{\mathrm{~d} x}=P(x) y+Q(x) y^{n} \quad(n \neq 0,1) .
$$

Equation (1) was named Bernoulli Equation. In 1696, Leibniz pointed out that Bernoulli Equation can be integrated. As a special kind of first order nonlinear ordinary differential equation, Bernoulli Equation have very extensive applications in mechanical engineering, etc. The study of Bernoulli Equation has important theoretical significance and extensive application value.

In 1985, Professor Cong Deng extended the definition of Bernoulli Equation in [1], in which an ample condition for the equation

$$
\frac{\mathrm{d} y}{\mathrm{~d} x}=P(x) f(y)+Q(x) g(y)
$$

to be integrated was proposed, and the general integral of (2) meeting requirement

$$
g(y) \frac{\mathrm{d}}{\mathrm{d} y}[f(y) / g(y)]=\alpha
$$

$(\alpha \neq 0$ is a constant) is follow

$$
\begin{aligned}
\frac{f(y)}{g(y)}= & \left.\exp \left[\alpha \int P(x) \mathrm{d} x\right]\right\} C+ \\
& \left.+\alpha \int Q(x) \exp \left[-\alpha \int P(x) \mathrm{d} x\right] \mathrm{d} x\right\},
\end{aligned}
$$

$C$ is a arbitrary constant.
Prof. Deng proposed that (2) with the requirement (3) be named Generalized Bernoulli Equation, and gave its general integral. Obviously, Bernoulli Equation is special example of (2) while $f(y)=y, g(y)=y^{n}, \alpha=1-n$.

In 2002, Prof. Deng discussed some literature about extension of Bernoulli Equation and proposed two questions in [2], one of is: if $g(y) \mathrm{d}(f(y) / g(y)) / \mathrm{d} y=H(y)$ isn't constant, can (2) be integrated to some special function $H(y)$ ? He thought that when we got some result in that two questions, we should extend the definition of Bernoulli Equation really and essentially. Another question is: when the condition (3) was founded, can the equation

$$
\frac{\mathrm{d} y}{\mathrm{~d} x}=P(x) h(y)+Q(x) f(y)+R(x) g(y)
$$

be integrated for some special $R(x)$ or $h(\mathrm{y})$ ? This question was discussed in [3] by Prof. Deng.

In 2010, Professor Ailian Hu extended Bernoulli Equation to a kind of nonlinear equation

$$
\frac{\mathrm{d} y}{\mathrm{~d} x}=P(x) f(y) \int \frac{\mathrm{d} y}{f(y)}+Q(x) f(y)\left[\int \frac{\mathrm{d} y}{f(y)}\right]^{n},
$$

and got its elementary integral method (1/f(y) can be integrated) in [4].

In fact, (5) is still Generalized Bernoulli Equation named by Prof. Deng in 1985.

$$
\begin{gathered}
\text { Let } F(y)=f(y) \int \frac{\mathrm{d} y}{f(y)}, \quad G(y)=f(y)\left[\int \frac{\mathrm{d} y}{f(y)}\right]^{n}, \text { then } \\
\frac{F(y)}{G(y)}=\left[\int \frac{\mathrm{d} y}{f(y)}\right]^{1-n}, \\
\frac{\mathrm{d}}{\mathrm{d} y}\left[\frac{F(y)}{G(y)}\right]=(1-n)\left[\int \frac{\mathrm{d} y}{f(y)}\right]^{-n} \frac{1}{f(y)}, \\
G(y) \frac{\mathrm{d}}{\mathrm{d} y}\left[\frac{F(y)}{G(y)}\right]=1-n .
\end{gathered}
$$

$1-n$ is a constant. 
In the following, we answer the first question of Prof. Deng. We first give a theorem, and then give its proofs, and then give two examples in the last part.

\section{THEOREM}

Equation (2) can be integrated, if real constants $\alpha, \beta$ meet

$$
g(y) \frac{\mathrm{d}}{\mathrm{d} y}\left[\frac{f(y)}{g(y)}\right]=\alpha+\beta\left[\frac{f(y)}{g(y)}\right] .
$$

If $\beta$ isn't 0 , the general integral of Equation (2) is as follows:

$$
\begin{array}{r}
\frac{g(y)}{f(y)+(\alpha / \beta) g(y)}=\exp \left[\int(\alpha P-\beta Q) \mathrm{d} x\right]\{C- \\
\left.-\beta \int P \exp \left[\int(\beta Q-\alpha P) \mathrm{d} x\right] \mathrm{d} x\right\} .
\end{array}
$$

$C$ is a constant of integration.

If $\alpha \neq 0$ but $\beta=0$, then the general integral of (2) is (4).

\section{PROVE}

When both constants $\alpha$ and $\beta$ are $0, g(y) \equiv 0$ or $f(y) / g(y)$ is constant, (2) is separable variable equation. Therefore, it can be integrated.

Obviously, when $\beta=0$, (2) is Generalized Bernoulli Equation named by Prof. Deng. We have known it can be integrated and its general integral is (4).

Let $u=\frac{g(y)}{f(y)+(\alpha / \beta) g(y)}$ for $\beta \neq 0$, then

$$
\begin{gathered}
\frac{\mathrm{d} u}{\mathrm{~d} y}=\frac{(f+g \alpha / \beta) \mathrm{d} g / \mathrm{d} y-[\mathrm{d} f / \mathrm{d} y+(\alpha / \beta) \mathrm{d} g / \mathrm{d} y] g}{[f(y)+(\alpha / \beta) g(y)]^{2}}= \\
\frac{(\mathrm{d} g / \mathrm{d} y) f-(\mathrm{d} f / \mathrm{d} y) g}{[f+(\alpha / \beta) g]^{2}}= \\
\frac{-g^{2}}{[f+(\alpha / \beta) g]^{2}} \cdot \frac{(\mathrm{d} f / \mathrm{d} y) g-(\mathrm{d} g / \mathrm{d} y) f}{g^{2}}= \\
\frac{-g}{[f+(\alpha / \beta) g]^{2}} \cdot g \frac{\mathrm{d}}{\mathrm{d} y}\left(\frac{f}{g}\right)=\frac{-g}{[f+(\alpha / \beta) g]^{2}} \cdot(\alpha+\beta f / g)= \\
-\frac{\beta}{f+(\alpha / \beta) g}=-\beta u / g .
\end{gathered}
$$

Now, (2) become:

$$
\begin{aligned}
& \frac{\mathrm{d} u}{\mathrm{~d} x}=-\frac{\beta}{g} u[P(x) f(y)+Q(x) g(y)] \\
& \frac{\mathrm{d} u}{\mathrm{~d} x}=-\beta u\left[P(x) \frac{f}{g}+Q(x)\right]
\end{aligned}
$$

Because $f / g=(1 / u)-\alpha / \beta$, so the above equation is

$$
\begin{aligned}
& \frac{\mathrm{d} u}{\mathrm{~d} x}=-\beta u\left[P(x)\left(\frac{1}{u}-\frac{\alpha}{\beta}\right)+Q(x)\right], \\
& \frac{\mathrm{d} u}{\mathrm{~d} x}=[\alpha P(x)-\beta Q(x)] u-\beta P(x) .
\end{aligned}
$$

It is a linear equation and its general integral is

$$
\begin{aligned}
u=\exp \left[\int(\alpha P(x)-\beta Q(x)) \mathrm{d} x\right]\{C- \\
\left.\quad-\beta \int P(x) \exp \left[\int(\beta Q(x)-\alpha P(x)) \mathrm{d} x\right] \mathrm{d} x\right\}
\end{aligned}
$$

Then the theorem is proved.

We suggest the naming (2) for Bernoulli-Deng Equation when the requirement (6) $(\alpha \neq 0$ or $\beta \neq 0)$ was founded.

\section{EXAMPLES}

Example 1. Solve the equation:

$$
\frac{\mathrm{d} y}{\mathrm{~d} x}=\mathrm{e}^{-x-y}+\mathrm{e}^{x+y}+\mathrm{e}^{-x}+\mathrm{e}^{x}
$$

Solving: Change the equation into:

$$
\frac{\mathrm{d} y}{\mathrm{~d} x}=\mathrm{e}^{-x}\left(1+\mathrm{e}^{-y}\right)+\mathrm{e}^{x}\left(1+\mathrm{e}^{y}\right) .
$$

Let

$P(x)=\mathrm{e}^{-x}, \quad Q(x)=\mathrm{e}^{x}, \quad f(y)=1+\mathrm{e}^{-y}, \quad g(y)=1+\mathrm{e}^{y}$,

then

$$
\begin{aligned}
g(y) \frac{\mathrm{d}}{\mathrm{d} y}\left[\frac{f(y)}{g(y)}\right] & =\left(1+\mathrm{e}^{y}\right) \frac{\mathrm{d}}{\mathrm{d} y}\left(\frac{1+\mathrm{e}^{-y}}{1+\mathrm{e}^{y}}\right)=\left(1+\mathrm{e}^{y}\right) \frac{\mathrm{d}}{\mathrm{d} y} \mathrm{e}^{-y}= \\
& -\mathrm{e}^{-y}-1=-1-\frac{f(y)}{g(y)} .
\end{aligned}
$$



is

This is a Bernoulli-Deng Equation and its general integral

$$
\frac{1+\mathrm{e}^{y}}{2+\mathrm{e}^{-y}+\mathrm{e}^{y}}=\exp \left(\mathrm{e}^{-x}+\mathrm{e}^{x}\right)\left[C+\int \exp \left(-x-\mathrm{e}^{x}-\mathrm{e}^{-x}\right) \mathrm{d} x\right]
$$

$C$ is a arbitrary constant.

Example 2. Solve the equation:

$$
\frac{\mathrm{d} y}{\mathrm{~d} x}=(x+y)(x+2)(y+2) \text {. }
$$

Solving: Change the equation into:

$$
\frac{\mathrm{d} y}{\mathrm{~d} x}=(x+2) y(y+2)+x(x+2)(y+2) .
$$

Let

$$
\begin{aligned}
& P(x)=x+2, \quad Q(x)=x^{2}+2 x, \\
& f(y)=y(y+2), \quad g(y)=y+2,
\end{aligned}
$$

then

$$
g(y) \frac{\mathrm{d}}{\mathrm{d} y}\left[\frac{f(y)}{g(y)}\right]=(y+2) \frac{\mathrm{d} y}{\mathrm{~d} y}=y+2=2+\frac{f(y)}{g(y)}
$$

This is a Bernoulli-Deng Equation and its general integral is

$$
\begin{aligned}
& \frac{y+2}{y^{2}+4 y+4}=\exp \left(4 x-\frac{x^{3}}{3}\right)\left[C-\int(x+2) \exp \left(\frac{x^{3}}{3}-4 x\right) \mathrm{d} x\right] \\
& \frac{1}{y+2}=\exp \left(4 x-\frac{x^{3}}{3}\right)\left[C-\int(x+2) \exp \left(\frac{x^{3}}{3}-4 x\right) \mathrm{d} x\right], \\
& y=\frac{\exp \left(\frac{x^{3}}{3}-4 x\right)}{C-\int(x+2) \exp \left(\frac{x^{3}}{3}-4 x\right) \mathrm{d} x}-2 .
\end{aligned}
$$

$C$ is a arbitrary constant.

\section{REFERENCES}

[1] Deng Cong. "Extension about Bernoulli Equation", in Journal of Zhaotong Teacher's College, pp.21-25.1985.

[2] Deng Cong. "Comment on Extension about Bernoulli Equation", in Journal of Zhaotong Teacher's College, vol.24, pp.52-54. October 2002.
[3] Deng Cong, and Wu Wenliang. "Integrable Circumstances of a Class of Nonlinear Ordinary Differential Equation", in Journal of Zhaotong Teacher's College, vol. 29, pp.1-5.October 2007.

[4] Hu Ailian. "Quasi-Bernoulli Equations and Their solving Methods", in

[5] Journal of Kashgar Teachers College, vol. 31, May 2010. 